\title{
Graphene oxide modified with PMMA via ATRP as a reinforcement filler
}

\author{
Gil Gonçalves, ${ }^{a}$ Paula A. A. P. Marques, ${ }^{* a}$ Ana Barros-Timmons, ${ }^{b}$ Igor Bdkin, ${ }^{a}$ Manoj K. Singh, ${ }^{a}$ \\ Nazanin Emami ${ }^{c}$ and José Grácio ${ }^{a}$
}

\author{
Received 31st May 2010, Accepted 26th July 2010 \\ DOI: $10.1039 / \mathrm{c0jm01674h}$
}

\begin{abstract}
Graphene is a two-dimensional new allotrope of carbon, which is stimulating great curiosity due to its superior mechanical, electrical, thermal and optical properties.

Particularly attractive is the availability of bulk quantities of graphene $(\mathrm{G})$ which can be easily processed by chemical exfoliation, yielding graphene oxide (GO). The resultant oxygenated graphene sheets covered with hydroxyl, epoxy and carboxyl groups offer tremendous opportunities for further functionalization opening plenty of opportunities for the preparation of advanced composite materials. In this work poly(methyl methacrylate) (PMMA) chains have been grafted from the GO surface via atom transfer radical polymerization (ATRP), yielding a nanocomposite which was soluble in chloroform. The surface of the PMMA grafted GO (GPMMA) was characterized by AFM, HRTEM, Raman, FTIR and contact angle. The interest of these novel nanocomposites lies in their potential to be homogenously dispersed in polymeric dense matrices and to promote good interfacial adhesion, of particular relevance in stress transfer to the fillers. PMMA composite films were prepared using different percentages of GPMMA and pristine GO. Mechanical analysis of the resulting films showed that loadings as low as $1 \%(\mathrm{w} / \mathrm{w})$ of GPMMA are effective reinforcing agents, yielding tougher films than pure PMMA films and even than composite films of PMMA prepared with GO. In fact, addition of $1 \%(\mathrm{w} / \mathrm{w})$ of GPMMA fillers led to a significant improvement of the elongation at break, yielding a much more ductile and therefore tougher material. Thermal analysis showed an increase of the thermal stability properties of these films providing evidence that strong interfacial interactions between PMMA and GPMMA are achieved. In addition, AFM analysis, in friction force mode, is demonstrated to be an effective tool to analyse the surface filler distribution on polymer matrices.
\end{abstract}

\section{Introduction}

Graphene is one of the most exciting materials being investigated today for fundamental studies as well as for potential applications. Pristine graphene is an atomically thin two-dimensional honeycomb material, however multiple layered graphene sheets are also of great interest. ${ }^{\mathbf{1} 2}$ For this reason the preparation of large quantities of different types of graphene and their characterization is receiving much attention. ${ }^{3}$ Graphene can be obtained by the exfoliation of graphite yielding well separated 2-D aromatic sheets which consist of $\mathrm{sp}^{2}$-bonded carbon atoms. ${ }^{4}$ Graphene sheets offer extraordinary electronic, thermal and mechanical properties and are expected to find a variety of applications such as in sensors, batteries, supercapacitors, hydrogen storage and reinforcing agents. ${ }^{5}$ One possible route to harnessing these properties for applications would be the incorporation of graphene sheets into a composite material. Nanocomposites incorporating graphene have the potential to rival or even surpass the performance of their carbon nanotube-based counterparts provided that cheap, large-scale production and processing methods for graphene become available. ${ }^{6}$ An effective

${ }^{a}$ TEMA-NRD, Mechanical Engineering Department, University of Aveiro, 3810-193 Aveiro, Portugal. E-mail: paulam@ua.pt; Fax: +351 234370 953; Tel: +351234370830

${ }^{b}$ CICECO, Department of Chemistry, University of Aveiro, 3810-193 Aveiro, Portugal; Fax: + 351234370 084; Tel: + 351234401525

${ }^{c}$ Department of Applied Physics and Mechanical Eng, Luleå University of Technology, Sweden approach in this regard is based on the chemical exfoliation of graphite to GO. ${ }^{7}$ This material consists of graphene derived sheets, heavily oxygenated, bearing hydroxyl, epoxide, carbonyl and carboxylic functional groups. ${ }^{8}$ The high density of oxygen functionalities at the GO sheet surface makes them highly hydrophilic and very stable aqueous dispersions can be obtained. ${ }^{7,9}$ However, aqueous dispersions are of limited interest in many applications. This is especially true regarding the preparation of polymer based nanocomposites, since most polymers are only soluble in organic solvents. In fact, recent works show an increasing interest in the dispersion of graphene in organic solvents. ${ }^{10,11}$

In the present work, the surface of GO was functionalized with an atom transfer polymerization initiator (ATRP) and subsequently PMMA chains were grown via living/controlled polymerization to yield a nanocomposite soluble in chloroform. Polymerizations from surfaces, also known as SIP (surface initiated polymerization), have been widely used in the preparation of nanocomposites. ${ }^{12}$ In this context, living/controlled radical polymerization mechanisms, such as ATRP, offer the possibility of preparing multifunctional materials with good control over the polymer molecular weight, polydispersity index, composition and end group functionality. ${ }^{13}$ Recently Lee et al. ${ }^{14}$ have also demonstrated the effectiveness of the ATRP strategy to grow styrene, methyl methacrylate, or butyl acrylate directly from the surface of GO without damaging the GO structure.

The surface modification of GO with PMMA chains aimed at increasing the compatibility between nano-sheets and polymer 
matrices as surface characteristics are determinant to yield nanocomposites with improved properties. Indeed, polymer nanocomposites have received much attention due to their enhanced mechanical, thermal, optical and barrier properties in comparison to pure polymer and/or micron-size composites. ${ }^{15}$ Some researchers also mention that the use of nanosized fillers has the potential to fundamentally improve the general performance of thermoplastic polymers. ${ }^{16,17}$ In fact, it has been shown that a considerable improvement in polymer nanocomposite mechanical properties can be achieved by adding nanosized fillers, such as $\mathrm{SiO}_{2}$ and $\mathrm{ZnO}$, at low volume content, i.e. in the range $1-5 \mathrm{vol} \%,{ }^{18-20}$ which is very important due to the high cost of nanosized fillers. However, when nanoparticles are used as fillers to prepare polymer nanocomposites, a homogeneous dispersion in the matrix is required. As the nanoparticles tend to aggregate, special techniques have to be developed to achieve homogeneous dispersions. One solution is mechanical mixing using high shear forces for dis-aggregation, whereas another technique is ultrasonic irradiation. Chemical methods can also be used, such as sol-gel processing ${ }^{21,22}$ or surface modification ${ }^{23-25}$ of nanoparticles to increase the compatibility between the nanoparticles and the organic phase. This opens a new way to prepare high performance polymeric materials, as was demonstrated by Fang et al. ${ }^{26}$ who functionalized GO with polystyrene (PS) chains and used these as reinforcement of the PS matrix. They found that both thermal and mechanical properties were improved relative to the pure polymer.

In this work, small percentages of previously modified GO, with PMMA chains via ATRP, were blended in a PMMA matrix. The effect of GO grafting with PMMA on the ensuing nanocomposite properties was studied by TGA and uniaxial tensile tests and complemented by AFM studies in friction mode. This, together with nanoindentation tests, provided further information to that previously reported in the literature.

\section{Experimental methods}

\section{Materials}

All chemicals were of analytical grade and used as received unless otherwise stated, except for the monomer (methyl methacrylate) which was passed through a column of alumina (Merck, 70-230 mesh) before use.

\section{GO preparation}

GO nanosheets were prepared by chemical exfoliation of graphite following the method described in a previous work of ours. ${ }^{27}$ Basically it consists of the chemical exfoliation of graphite in a strong oxidant medium (conc. $\mathrm{H}_{2} \mathrm{SO}_{4}$ and $\mathrm{KMnO}_{4}$ ) under vigorous stirring. The resultant suspension was extensively washed with distilled water by filtration and subsequent centrifugation in order to remove residual unexfoliated graphite and oxidizing agents. The resulting GO was lyophilised to prevent agglomeration.

\section{GO nanosheets functionalization with PMMA}

This procedure involved a three-step reaction pathway as illustrated in Scheme 1.

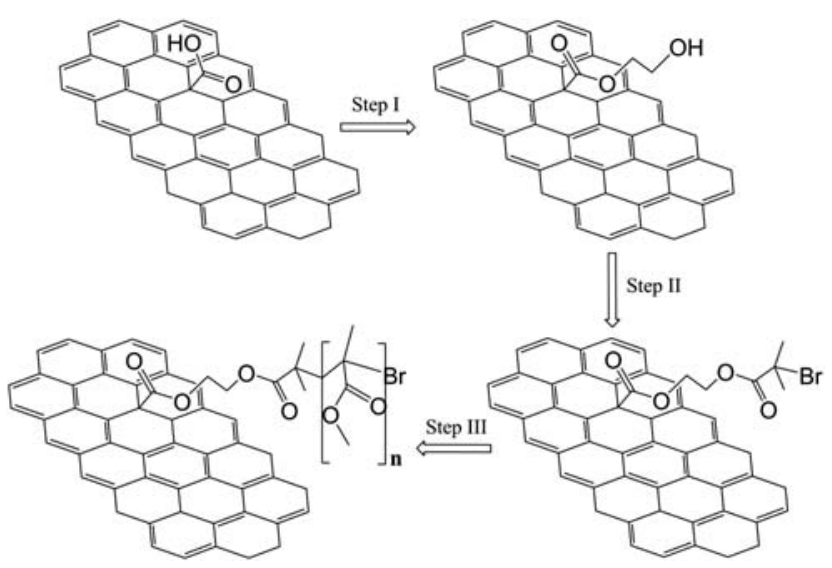

Scheme 1 Reaction pathway involved in the functionalization of GO nanosheets with polymer chains.

Step I - GO (200 mg) was suspended in $\mathrm{SOCl}_{2}(20 \mathrm{~mL})$ and stirred for $24 \mathrm{~h}$ at $65^{\circ} \mathrm{C}$ in a round-bottom flask equipped with a condenser, $\mathrm{N}_{2}$ inlet and magnetic bar. Upon this, excess $\mathrm{SOCl}_{2}$ was removed by successive centrifugation/redispersion using THF. The resulting solid was suspended in ethylene glycol $(20 \mathrm{~mL})$ in a round-bottom flask equipped with a condenser and the suspension was stirred for $48 \mathrm{~h}$ at $120^{\circ} \mathrm{C}$. As done previously, unreacted ethylene glycol was removed by successive centrifugation/redispersion using THF. The solid was then dried under vacuum to yield hydroxyl functionalized graphene $(\mathrm{G}-\mathrm{OH})$.

Step II - G-OH (200 mg) was dispersed in $\mathrm{CHCl}_{3}(15 \mathrm{~mL})$ in a round-bottom flask. The suspension was purged with $\mathrm{N}_{2}$ for one hour and then, triethylamine $(1.64 \mathrm{mmol})$ and $4-$ dimethylaminopyridine $(1.64 \mathrm{mmol})$ were added and the mixture was stirred for $1 \mathrm{~h}$. Next, the ATRP initiator (2-bromo-2methylpropionyl bromide) was added $(13.0 \mathrm{mmol})$ and the mixture was stirred for $48 \mathrm{~h}$ at room temperature. The resulting ATRP modified graphene (G-Br) was purified by successive centrifugation/redispersion using $\mathrm{CHCl}_{3}$.

Step III - G-Br (200 mg) was dissolved in DMF (6 mL) and $\mathrm{CuBr}(0.27 \mathrm{mmol})$ and PMDETA $(0.27 \mathrm{mmol})$ were added. Upon purging the reaction mixture with $\mathrm{N}_{2}$ for 30 min, methyl methacrylate $(28 \mathrm{mmol})$, was added and the temperature was raised to $65^{\circ} \mathrm{C}$. The polymerization was carried out for $24 \mathrm{~h}$. The graphene/PMMA nanocomposite (GPMMA) was isolated by three successive centrifugation/redispersion using $\mathrm{CHCl}_{3}$. All the modification steps were monitored by solubility tests.

\section{Hydrolysis of PMMA from GPMMA}

GPMMA (20 mg) was dissolved in THF $(3 \mathrm{~mL})$ with $8 \mathrm{mg}$ of $\mathrm{KOH}$ and the reaction mixture was stirred for $10 \mathrm{~h}$ at $50^{\circ} \mathrm{C}$ under a nitrogen atmosphere, following a method described elsewhere. ${ }^{28}$ The resultant solution was filtered through a $0.3 \mu \mathrm{m}$ Teflon filter to remove the graphene sheets and analysed by GPC.

\section{Preparation of PMMA/GPMMA films}

The preparation of PMMA reinforced films was based on the solvent casting method. First, specific amounts of the functionalized graphene sheets were dispersed in chloroform by 
sonication during $30 \mathrm{~min}$ at room temperature and then PMMA $(30 \mathrm{wt} \%)$ was dissolved in the same medium. The resultant mixtures were slowly poured onto Teflon plates and dried at $85^{\circ} \mathrm{C}$ overnight. Finally, the dried films were removed from the Teflon plates to yield PMMA/GPMMA films with an average thickness of $0.065 \pm 0.002 \mathrm{~mm}$. These films were stored at $25^{\circ} \mathrm{C}$ and $40 \%$ humidity before analysis.

\section{Characterization}

The thermogravimetric (TGA) assays were carried out in a Shimadzu TGA 50 analyzer equipped with a platinum cell. The samples were heated at a constant rate of $10{ }^{\circ} \mathrm{C} \mathrm{min}{ }^{-1}$ from room temperature to $600{ }^{\circ} \mathrm{C}$, under $\mathrm{N}_{2}$ flow.

Ultra-high resolution analytical scanning electron microscope HR-FESEM Hitachi SU-70 was used to observe the samples. Conventional high resolution TEM (JEOL 2200F TEM/STEM) operated at $200 \mathrm{kV}$ with a point resolution of $0.16 \mathrm{~nm}$, equipped with a GIF-2000 spectrometer was also used. The samples for TEM analysis were prepared by depositing an aliquot of the GO and G-PMMA suspension onto a carbon grid and then the solvent was allowed to evaporate.

The FTIR spectra were recorded from $\mathrm{KBr}$ pellets (Aldrich, 99\%, FT-IR grade) using a Mattson 7000 FT-IR spectrometer with resolution 8 and 256 interferograms.

A digital instruments MultiMode Scanning Probe Microscope (SPM) with a Nanoscope IIIA controller in contact friction mode was used for the AFM measurements.

FT-Raman spectra were recorded using a Bruker RFS100/S FT-Raman spectrometer (Nd:YAG laser, $1064 \mathrm{~nm}$ excitation).

For SEC analysis, $5 \mathrm{mg}$ of polymer was dissolved in $1000 \mu \mathrm{L}$ of DMA solution at $20^{\circ} \mathrm{C}$ for $30 \mathrm{~min}$ and further filtered through a $0.3 \mu \mathrm{m}$ filter yielding a sample concentration of circa $0.5 \%$ $\left(5 \mathrm{mg} \mathrm{mL}^{-1}\right)$. The SEC analysis was carried out on two PLgel $10 \mathrm{~mm}$ MIXED B $300 \times 7.5 \mathrm{~mm}$ columns protected by a PLgel $10 \mathrm{~mm}$ pre-column (Polymer Laboratories, UK) using a PL-GPC 110 system (Polymer Laboratories, UK). The columns, injector system and the detector (RI) were maintained at $70{ }^{\circ} \mathrm{C}$ during the analysis. The eluant (DMA) was pumped at a flow rate of $0.9 \mathrm{~mL}$ $\mathrm{min}^{-1}$. The analytical columns were calibrated with polystyrene standards (Polymer Laboratories, UK) in the range 1.7-100.0 $\mathrm{kDa}$. The injected volume was $100 \mu \mathrm{L}$.

Tensile properties of the films were measured on a texture analyser (model TA.Hdi, Stable Micro Systems, England) equipped with fixed grips lined with thin rubber on the ends. The specimens were cut into $10 \mathrm{~mm}$ wide and $90 \mathrm{~mm}$ long strips. The initial grip length was $50 \mathrm{~mm}$ and the specimens were uniaxially stretched at a constant crosshead speed of $0.5 \mathrm{~mm} \mathrm{~s}{ }^{-1}$. All experiments were conducted at $25 \pm 2{ }^{\circ} \mathrm{C}$ and $50 \pm 2 \% \mathrm{RH}$. At least, ten samples of each film type were tested. Young's modulus (E), percentage elongation or strain at break and tensile strength or stress at break, were determined from stress-strain curves obtained from tensile tests.

Nanoindentation tests were performed using the CSM Nanoindenter with a Berkovich diamond tip. The hardness and elastic modulus of nanocomposites with different graphene percentages were measured as a function of indentation depth (20 tests for each sample).
The typical nanoindentation test consists of applying a force of $1 \mathrm{mN}$ at a loading/unloading rate of $2 \mathrm{mN} \mathrm{min}^{-1}$ with a pause of $2 \mathrm{~s}$ at maximum force. The hardness and elastic modulus were obtained from the curves using the Oliver-Pharr method. ${ }^{29}$

Water contact angles (CA) were measured using a "surface energy evaluation system" commercialized by Brno University (Czech Republic). Each $\theta$ value was the average of five determinations.

\section{Results and discussion}

\section{Graphene oxide functionalization with PMMA}

The prepared aqueous dispersion of GO was characterized to confirm the presence of well exfoliated GO sheets as described by the present authors in a previous publication. ${ }^{27}$ The GO solution was then freeze dried by lyophilisation to obtain a feathery powder.

Taking advantage of the oxygen functionalities of the asprepared GO nanosheets, the functionalization with polymer chains via ATRP was carried out. Following the procedures described in the literature, ${ }^{30-32}$ the free carboxylic acid groups of GO were converted to acyl chloride groups, which upon treatment with ethylene glycol, yielded graphene sheets rich in hydroxyl groups. In a second step, the ATRP initiator was grafted onto the $\mathrm{OH}$ rich graphene sheets and finally PMMA chains were grown via ATRP.

After surface modification of GO with PMMA the reaction mixture changed from yellow-brown to a black colour indicating the conversion of GO to form GPMMA. The suspension of GPMMA in the chloroform solution is shown in the inset of Fig. 1d, which is clearly darker than the GO/water solution (see the inset of Fig. 1a). For a detailed morphological study, TEM analysis of as synthesized GO and GPMMA was performed. Fig. 1a shows a general view of a GO nanosheet on the TEM carbon grid, clearly illustrating the flake-like shape of GO where the edges tend to fold and roll. Fig. $1 \mathrm{~b}$ shows a higher magnification of the area denoted by the square in Fig. 1a. The same image, after filtering in the frequency domain to remove unwanted noise (fast Fourier transform), is shown in Fig. 1c where the hexagonal graphene network can be observed. Fig. 1d depicts the TEM image of the GPMMA sample where some dark features can be observed. The high magnification image (Fig. 1e) of the area denoted by the square region in Fig. 1d, clearly shows these dark spots as opposed to what it observed for pristine GO (Fig. 1b). The presence of such spots can be attributed to polymer brushes and has been observed by other authors with different polymers as described elsewhere. ${ }^{33,34}$ According to Yang et al. ${ }^{33}$ in solution the grafted polymer chains are extended due to their solubility in the solvent, however, after drying the polymer chains collapse onto the surface of the GO sheets forming nanosized domains that correspond to the dark spots observed.

The surface of GPMMA was characterized by infrared spectroscopy and compared to GO (Fig. 2). The characteristic features of GO in the FTIR spectrum are the absorption bands corresponding to the $\mathrm{C}=\mathrm{O}$ stretching vibrations from carbonyl and carboxylic groups at $1720 \mathrm{~cm}^{-1}$, the $\mathrm{C}-\mathrm{OH}$ stretching at $1227 \mathrm{~cm}^{-1}$, and the $\mathrm{C}-\mathrm{O}$ vibrations of the epoxy groups at $c a$. $1139 \mathrm{~cm}^{-1}$ and $873 \mathrm{~cm}^{-1} \cdot{ }^{35,36}$ The spectrum also shows a band at 


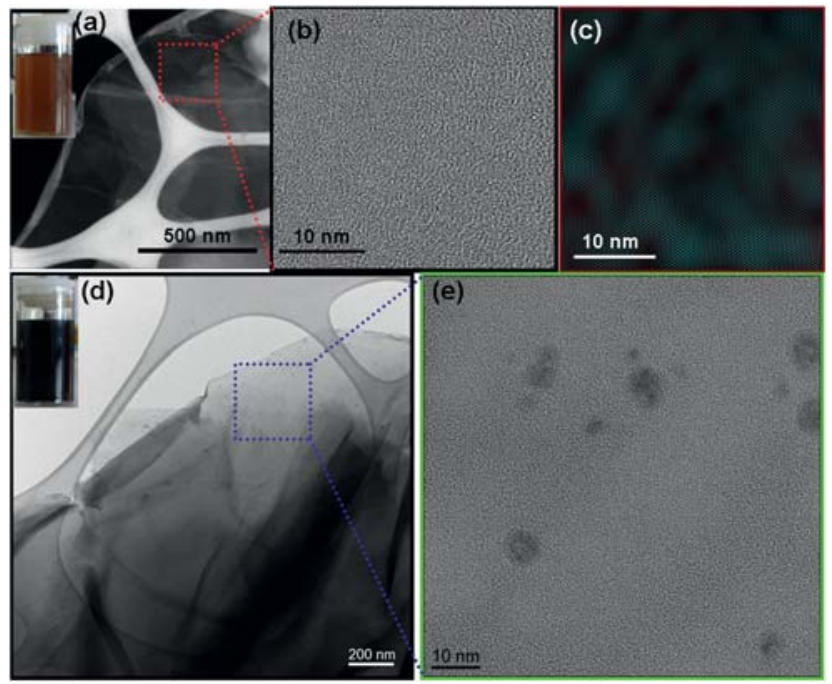

Fig. 1 TEM analysis of the synthesized GO and GPMMA. Fig. 1a shows bright field TEM image of the GO sheet on a TEM carbon grid. Figs. $1 \mathrm{~b}$ and $\mathrm{c}$ show HRTEM and its FFT image of GO respectively. Fig. 1d depicts the TEM image of the GPMMA sample, the high magnification image of the area denoted by the square region is shown in Fig. 1e. Insets in Fig. 1a and 1b show the photographic images of GO solutions in water and GPMMA in chloroform respectively.

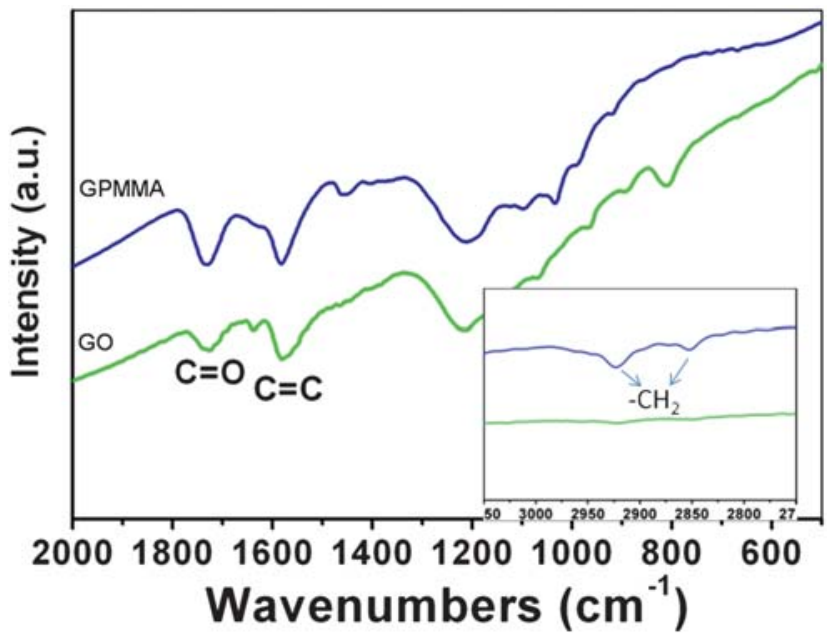

Fig. 2 FTIR spectra of (a) GO and (b) GPMMA.

$1620 \mathrm{~cm}^{-1}$, attributed to aromatic carbon double bonds. ${ }^{27}$ After functionalization with PMMA chains, the presence of the $\mathrm{C}=\mathrm{O}$ groups is not helpful to determine whether any significant modification had occurred as it was already present in GO. However, the increase of the intensity of the carbonyl band and the presence of characteristic bands of $-\mathrm{CH}_{2}$ at 2850 and $2920 \mathrm{~cm}^{-1}$ in the spectrum of GPMMA indicates the presence of the polymer chains. Raman spectroscopy was also used to assess the surface modification of GO. As expected, a decrease of intensity of the characteristic D $\left(1330 \mathrm{~cm}^{-1}\right)$ and $\mathrm{G}\left(1590 \mathrm{~cm}^{-1}\right)$ bands of graphene in the GPMMA spectrum was observed in relation to what was observed for $\mathrm{GO}$, which is associated with surface defects created during the surface treatments. ${ }^{34}$

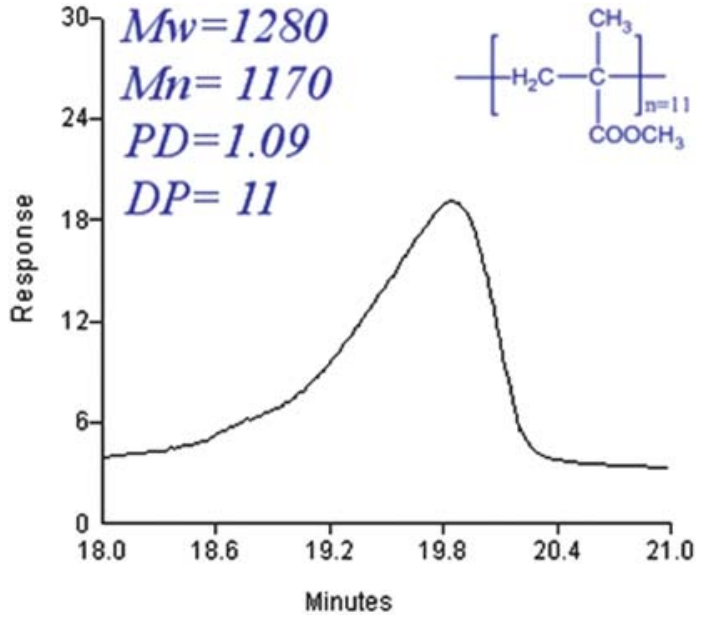

Fig. 3 GPC analysis of PMMA chains removed from the surface of GPMMA.

Contact angle measurements, using water, confirmed the significant difference of the surface characteristics upon PMMA grafting. For GO the water droplet was immediately adsorbed by the substrate whilst for GPMMA the water contact angle was $67.4^{\circ}( \pm 0.4)$ which is in agreement with the water contact angle of pure PMMA $\left(68^{\circ}\right){ }^{37}$

The chain length of PMMA (MW) grown from the surface of $\mathrm{GO}$, as well as its molecular weight distribution (MWD), was determined by GPC. Prior to this, the chains were removed from the surface via hydrolysis following Baskaran's work. ${ }^{28}$ The GPC obtained is illustrated in Fig. 3 which clearly proves that the polymerization is very well controlled, with a MWD (or PD) of 1.09. On the other hand, the degree of polymerization was only 11. This low MW suggests that surface modification with the ATRP initiator seems to be rather efficient with the various active sites competing for the available monomer.

The TGA curves are shown in Fig. 4 for GO and GPMMA. Despite the distinct characteristics between commercial PMMA and PMMA chains grown from GO, such as MW and MWD for example, for comparison purposes its degradation pattern under the same analyses conditions is also plotted. It is clear that the decomposition profile of GPMMA is rather different from those of GO and PMMA. The $25 \%$ weight loss between 176 and $247^{\circ} \mathrm{C}$

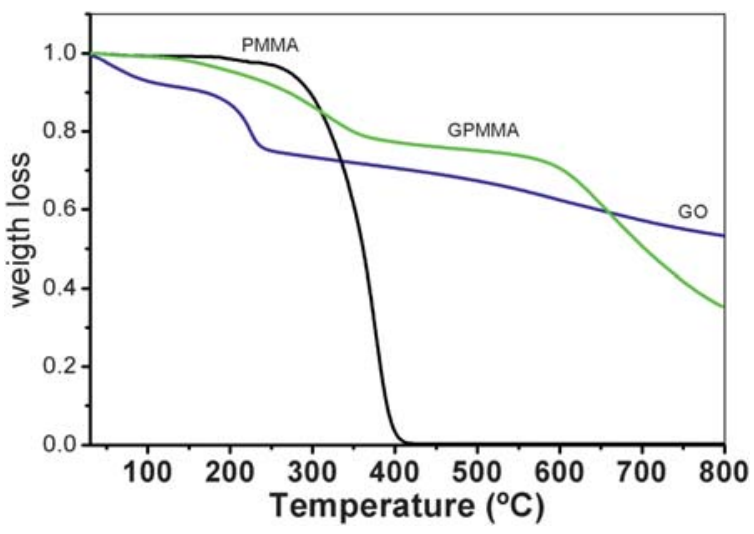

Fig. 4 The TGA curves of GO, PMMA and GPMMA. 
registered for $\mathrm{GO}$ is attributed to the decomposition of labile oxygen functional-containing groups. ${ }^{38}$ After that, GO is stable up to $500{ }^{\circ} \mathrm{C}$ when decomposition of the carbon structure occurs. As regards the GPMMA nanocomposite a $35 \%$ weight loss is observed between 145 and $397{ }^{\circ} \mathrm{C}$, whilst for the commercial PMMA sample thermal degradation only begins at $270{ }^{\circ} \mathrm{C}$. The difference between GPMMA and the commercial PMMA is thought to be due to the thermal decomposition of oxygencontaining groups present on the GPMMA surface resulting from previous surface treatments of the GO. Further differences are also observed at higher temperatures. Whilst the commercial PMMA sample is completely degraded at $400{ }^{\circ} \mathrm{C}$, the PMMA grafted on the $\mathrm{GO}$ surface is stable up to $560{ }^{\circ} \mathrm{C}$. The increase in thermal stability observed for the grafted PMMA, i.e. GPMMA may be ascribed to the ability of the graphene surface to capture free radicals generated during thermal decomposition.

To further understand the surface of GO and GPMMA and to obtain complementary information to the TEM analysis, AFM studies in friction mode were performed. It is known that the adhesion force $\left(\mathrm{F}_{\mathrm{ad}}\right)$ and the friction coefficient $(\mu)$ of a material depend on its atomic structure and can be calculated by the following equation $\mathrm{F}_{\mathrm{L}}=\mu\left(\mathrm{F}_{\mathrm{N}}+\mathrm{F}_{\mathrm{ad}}\right)$, where $\mathrm{F}_{\mathrm{L}}$ is the lateral force, also known as friction force, and $F_{N}$ the normal force. From the graphics inserted in Fig. 5 it is possible to see the different response of the materials by applying three different normal forces. The results show that the friction coefficients are relatively similar in both samples. However, the adhesion force is three times higher in the case of the $\mathrm{GO}$ sheets $\left(\mathrm{F}_{\mathrm{ad}} \mathrm{GO}=11.8\right.$ $\mathrm{nN}$ and $\left.\mathrm{F}_{\mathrm{ad}} \mathrm{GPMMA}=3.4 \mathrm{nN}\right)$. These results can be attributed to the different chemical surface of GPMMA which results in different atomic interactions with the AFM cantilever. Careful analysis of the graphs shows a linear relationship between the normal and lateral forces which point to a homogeneous distribution of the polymer chains at the GO surface. These results are in agreement with an efficient modification of the GO surface
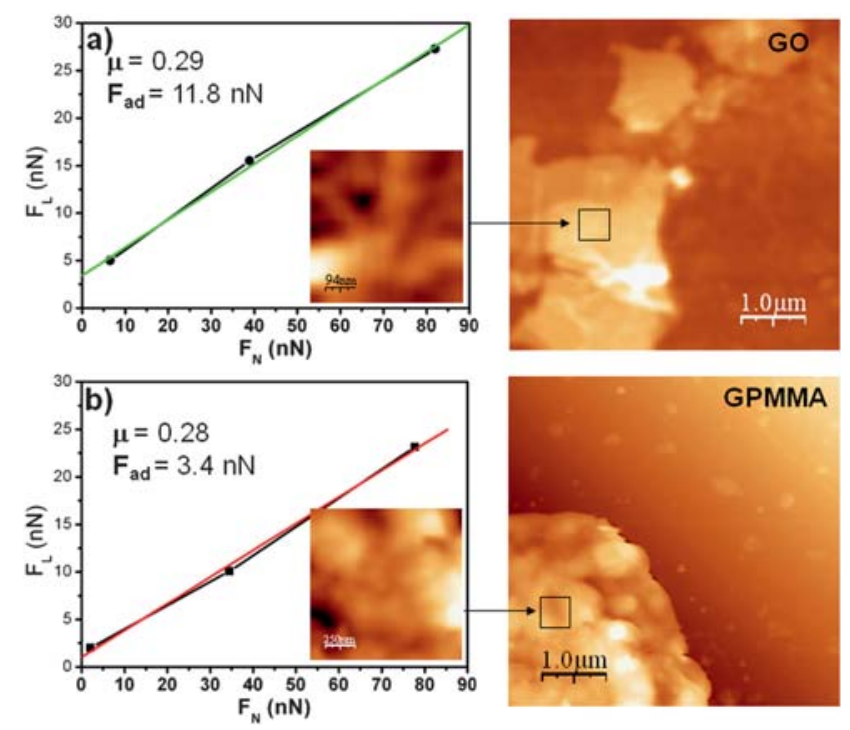

Fig. 5 A graphical representation of lateral versus normal forces determined by the AFM analysis in friction mode and contact-mode AFM topography images of (a) GO and (b) GPMMA. with the ATRP initiator and subsequent uniform polymerization, as discussed before.

\section{Nanocomposite films of PMMA prepared with GPMMA fillers (PMMA/GPMMA)}

To assess the compatibility between the previously prepared GPMMA nanosheets with PMMA matrix, nanocomposite films with $0.5,1$ and $3 \%(\mathrm{w} / \mathrm{w})$ of GPMMA dispersed in PMMA were prepared. A homogeneous dispersion of graphene sheet is observed for $1 \%(\mathrm{w} / \mathrm{w})$ GPMMA in a PMMA matrix (see Fig. 6a). This is in contrast with the $3 \%(w / w)$ GPMMA in a PMMA matrix, where graphene sheet agglomeration is clearly observed by SEM (see Fig. 6b).

PMMA films without fillers and PMMA films with 1\% (w/w) of GO were also prepared for comparison. During the preparation of the suspensions it was evident that the dispersion of GPMMA fillers in the PMMA solution was much better than that of GO. In fact, attempts to prepare a film with $3 \%(\mathrm{w} / \mathrm{w})$ of $\mathrm{GO}$ yielded heterogeneous films. The films obtained after solvent evaporation illustrate these observations as shown in the photographs of Fig. 7a. For the same filler load [1\%(w/w)] the difference in the dispersion behaviour is evident. When GPMMA was used, a much more homogeneous distribution of the fillers in the PMMA matrix was achieved, even at 3\% (w/w).

AFM analysis in the friction force mode was used to characterize these films. Fig. 7b shows the 3-D topography image (vertical scale) of the film prepared using GPMMA [1\% (w/w)] with surface roughness RMS of $100 \mathrm{~nm}$ at $\mathrm{F}_{\mathrm{N}}=80 \mathrm{nN}$. As conventional topography AFM images did not provide distinction between the fillers and the polymer matrix, AFM analyses in the friction force mode at different $F_{N}$ values proved to be a useful approach to investigate the distribution of graphene based fillers in the PMMA film. To the best of our knowledge, this was the first time this approach has been used for this type of materials. Whilst the PMMA matrix (region A) presents an average friction force of $22 \mathrm{nN}$, the GPMMA (region B) presents an average friction force of $42 \mathrm{nN}$. This difference can be justified by the lower atomic interaction between the AFM tip and the PMMA. ${ }^{39}$

From the dependence of friction force as a function of the applied normal force (graphical representation in Fig. 7c) the friction coefficients and adhesion forces for the PMMA (region A) and for the GPMMA (region B) can be determined. The adhesion forces $\left(\mathrm{F}_{\mathrm{ad}}\right)$ of the PMMA and GPMMA were found to

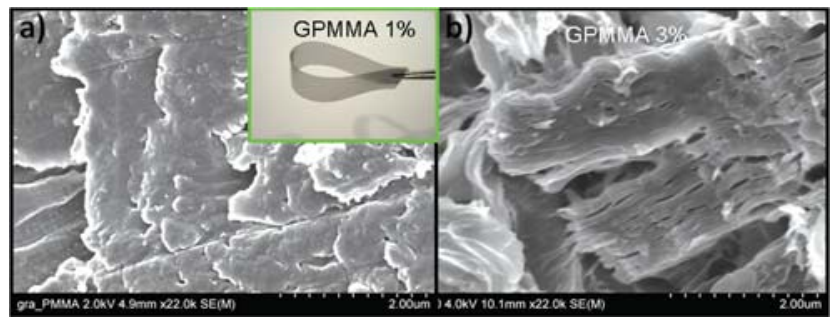

Fig. 6 SEM images of PMMA films prepared with the inclusion of $1 \%$ $(w / w)$ (a) and 3\% (w/w) (b) of GPMMA. The inset in (a) shows a photographic image of the $1 \%(\mathrm{w} / \mathrm{w})$ film illustrating the flexibility of this film. 

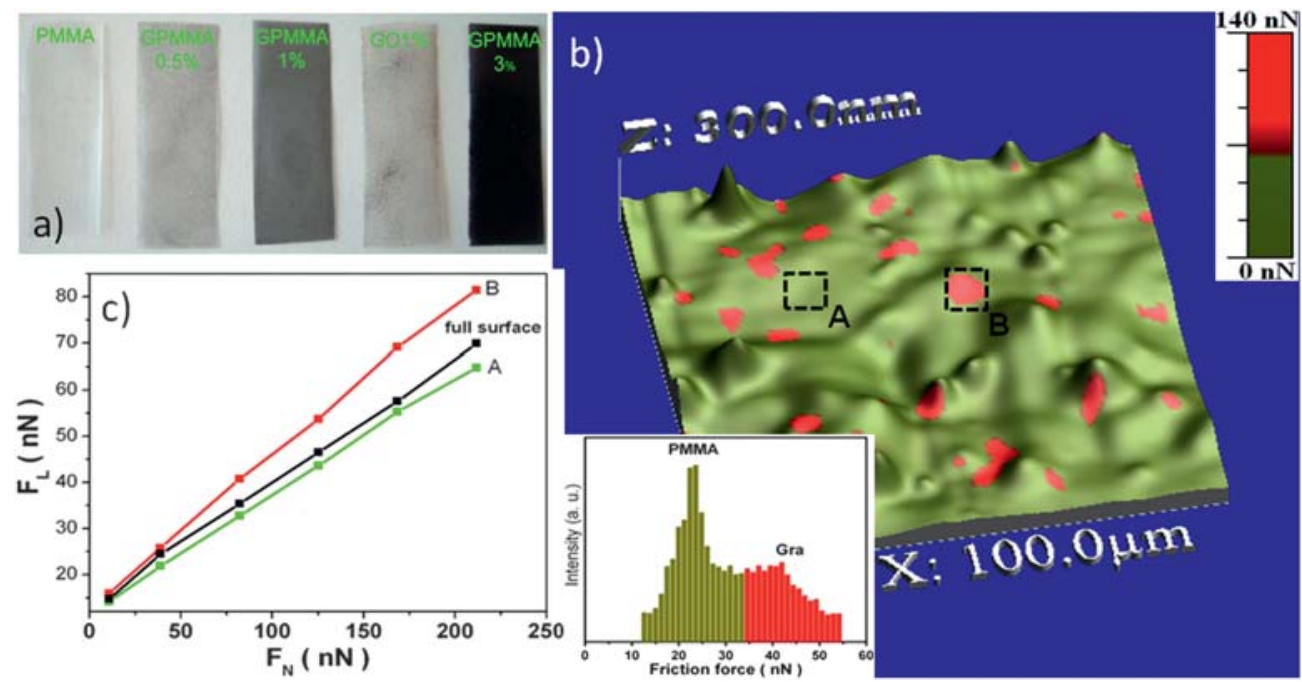

Fig. 7 (a) Photographs of the films prepared with and without fillers. (b) Topography and contact-mode friction AFM image of the PMMA-graphene composite with normal force $\sim 80 \mathrm{nN}$. Colour on this figure corresponds to values of friction force. Insert: histogram of the friction AFM image. (c) Plot of the friction force $\left(\mathrm{F}_{\mathrm{L}}\right)$ versus the applied load $\left(\mathrm{F}_{\mathrm{N}}\right)$ for the full surface, for area A (PMMA) and for area B (GPMMA) shown in image (b).

be 38.8 and $16.5 \mathrm{nN}$, respectively and the friction coefficients $(\mu)$ were 0.326 for PMMA and 0.516 for G-PMMA. The larger adhesion force obtained for PMMA may be explained by extra bending of the PMMA surface under AFM tip and, as a consequence, changing of effective contact area between the sample and the AFM tip. As regards the full surface area considered in Fig. $7 \mathrm{~b}$, it is possible to see that a rather small concentration of GPMMA $[1 \%(\mathrm{w} / \mathrm{w})]$ leads to an increase of the friction force. The measured value $\mu$ of $\sim 0.32$ for PMMA correlates well with data found in the literature. ${ }^{40}$

The mechanical behaviour of the composite films was studied by nanoidentation and uniaxial tensile tests. Regarding nanoindentation, the data recorded as a function of time for a single loading and unloading cycle curve provided information about the viscoelastic, elastic, and plastic deformation behaviour of nanocomposite films. ${ }^{41}$ Fig. 8 displays typical loading-unloading curves $^{42}$ for pure PMMA films and for the films prepared using $0.5,1$ and $3 \%(w / w)$ of GPMMA and $1 \%$ of GO which shows that

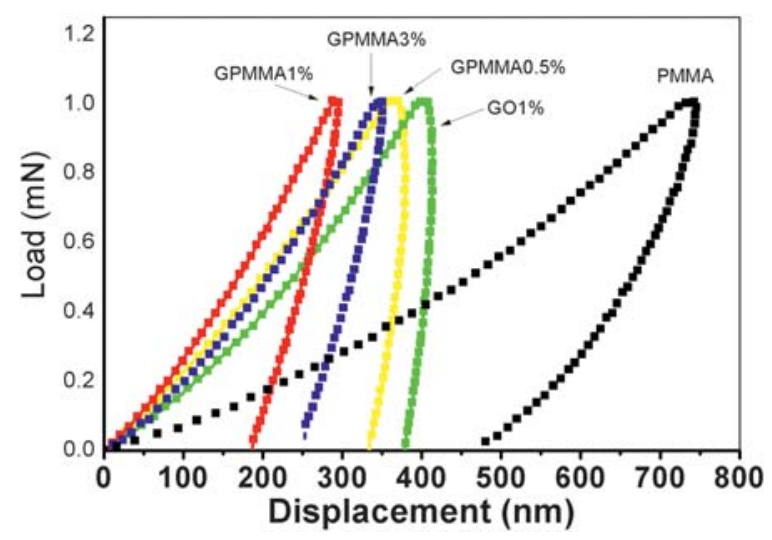

Fig. 8 Load-displacement nanoindentation curves of PMMA films with and without graphene fillers. incorporation of fillers into the PMMA matrix increases the resistance to penetration by the nanoindenter. For the pure PMMA film, the maximum indentation depth at the maximum load of $1.0 \mathrm{mN}$ was $760 \mathrm{~nm}$ which was reduced to $420 \mathrm{~nm}$ upon addition of $1 \%(\mathrm{w} / \mathrm{w})$ of GO. This reduction was even more significant for the series of films prepared with GPMMA fillers: $0.5 \%(\mathrm{w} / \mathrm{w})$, maximum depth $380 \mathrm{~nm} ; 3 \%(\mathrm{w} / \mathrm{w})$ maximum depth $350 \mathrm{~nm} ; 1 \%(\mathrm{w} / \mathrm{w})$, maximum depth $290 \mathrm{~nm}$. The results obtained for loads of $1 \%(\mathrm{w} / \mathrm{w})$ for GO and GPMMA show that the reinforcement effect of GPMMA is significantly more effective due to the surface modification and subsequent increased adhesion with the polymer matrix. Furthermore, the results obtained for the films prepared with GPMMA show that $1 \%(\mathrm{w} / \mathrm{w})$ loading yields the stiffest nanocomposite films. This may be associated with percolation effects at higher loadings.

For further confirmation uniaxial tensile tests were performed to assess the tensile properties of the composite films. A set of five measurements on five different strips was performed on each sample for statistical accuracy. The stress-strain curves of pure PMMA films as well as for films prepared using $0.5,1$ and $3 \%(\mathrm{w} / \mathrm{w})$ of GPMMA, and 1\% of GO are presented in Fig. 9. The tensile mechanical values are summarized in Table 1.

At very low stresses and strains, all the samples behave as a linear elastic solid with Young's modulus varying between 18.7 MPa for pure PMMA and 21.8 MPa for films prepared with $1 \%$ (w/w) of GPMMA (the highest value obtained). Films prepared with $1 \%(w / w)$ of GPMMA also showed plastic regime characterized by average values of uniform and ultimate elongations of $4.0 \%$ and $4.8 \%$ respectively for relatively high level of flow stress (42.0 MPa at break). It appears clear that for this condition the material exhibits elasto-plastic deformation and reasonable ductility.

Such mechanical improvements could be attributed to the efficient load transfer between graphene sheet and the PMMA matrix (see Fig. 6a). This kind of polymer matrix could be very interesting for load bearing applications. However, increasing 


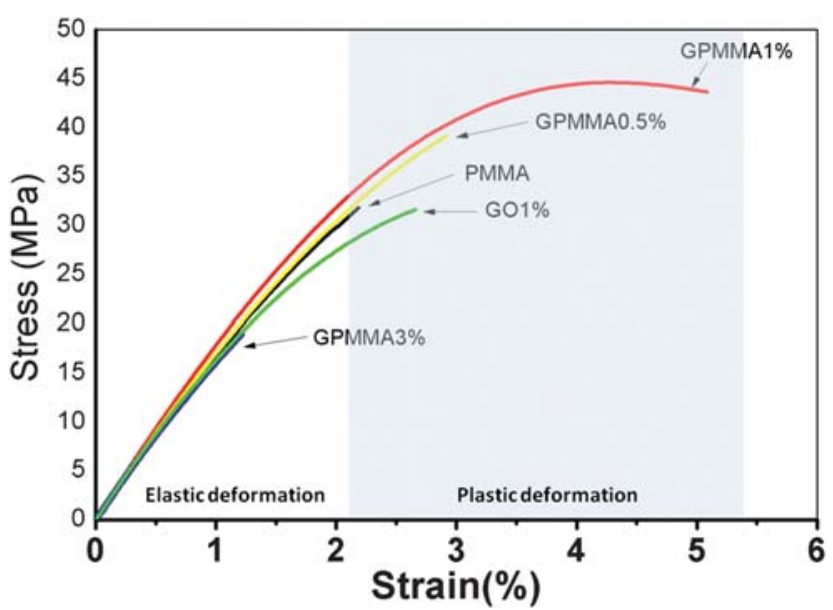

Fig. 9 Stress-strain dependencies of PMMA films with and without graphene fillers.

the concentration of GPMMA to about 3\% (w/w) deteriorated the mechanical behaviour of the overall composite film. This may be due to the agglomeration of graphene sheets creating defects in the PMMA matrix composite (see Fig. 6b). Similar downturns of the mechanical properties of composites containing polymergrafted MWCNTs at higher MWCNT loadings have been reported and attributed to aggregation and percolation effects. $^{43,44}$

Concerning the films prepared with $1 \%$ GO in the PMMA matrix, the Young's modulus, tensile strength and elongation at break values determined are comparatively lower than the ones obtained for $0.5 \%$, and $1 \%$ GPMMA in the PMMA matrix. This indicates that GO sheets do not establish appropriate interfaces with the PMMA matrix. So there might be a lack of load transfer from matrix to GO sheets which directly reflects the mechanical behaviour of the overall composite. Young's modulus and tensile strength values for the film with $1 \% \mathrm{GO}$ are even smaller than the values obtained for PMMA film prepared without fillers. Moreover, the elongation at break for $1 \%$ GO in the PMMA matrix is $2.7 \%$, which is slightly higher than pure PMMA (2.3\%). This may be due to the presence of graphene sheets which are known for their elasto-plastic behaviour. ${ }^{45-48}$

Finally, the thermal stability of the as-prepared films was evaluated by thermogravimetric analysis under a nitrogen atmosphere; the curves are shown in Fig. 10. Weight loss curve profiles are very similar for all the films; however the onsets of the thermal decomposition of the films with GPMMA were about $50{ }^{\circ} \mathrm{C}$ higher than that for pure PMMA. There is also a slight improvement of the thermal stability for the samples containing GPMMA, even for loads as low as $0.5 \%(\mathrm{w} / \mathrm{w})$. As discussed previously, the graphene fillers may restrain the attack of free radicals generated during the thermal decomposition of PMMA. For the films prepared with GPMMA, this effect is more pronounced when compared with films prepared with GO since the PMMA chains are more strongly mixed with the fillers in the first case, facilitating the radical capture.

\section{Conclusions}

In summary we have successfully modified the surface of GO with PMMA chains $(\mathrm{PD}=1.09)$ via ATRP. This strategy can be further exploited to grow a large range of polymers from the GO surface only by changing the monomer. The resulting nanocomposites were readily dispersed in organic solvents and used as reinforcement fillers in the preparation of PMMA composite films. When GPMMA was used, a much more homogeneous distribution of the fillers in the PMMA matrix was achieved.

Mechanical analysis of the resulting films showed that loadings as low as $1 \%(\mathrm{w} / \mathrm{w})$ of GPMMA are effective reinforcing agents, yielding tougher films than those of pure PMMA. Addition of $1 \%(w / w)$ of GPMMA fillers clearly led to a significant improvement of the elongation at break, yielding a much more ductile and, therefore, tougher material.

Thermal analysis showed an increase in the thermal stability properties of the films prepared with GPMMA fillers in comparison with non modified GO fillers providing evidence that strong interfacial interactions between PMMA and GPMMA are achieved.

We have also demonstrated that AFM analysis in the friction force mode can be an effective tool to analyse the surface filler distribution on the polymer matrices. In this particular case, the distribution of graphene fillers was detected on the surface of the film by differences in the friction forces.

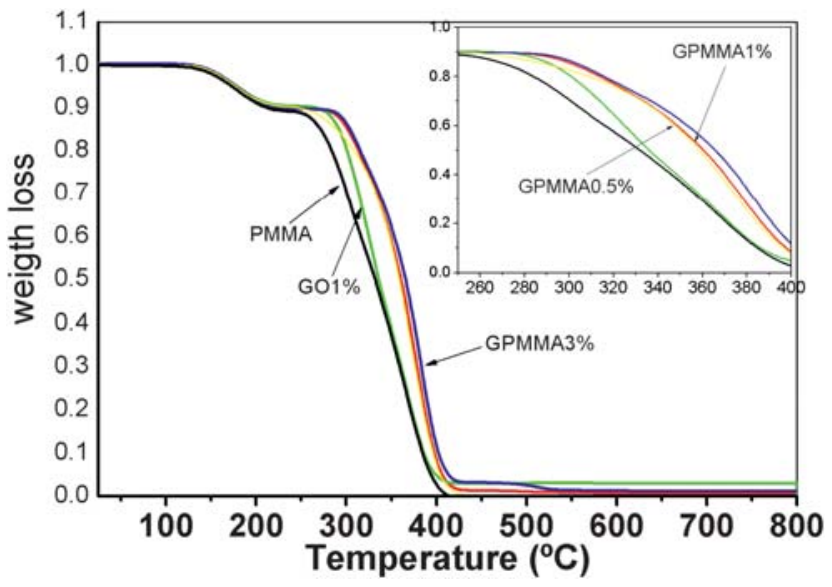

Fig. 10 The thermal decomposition of the pure PMMA and PMMA/ GPMMA films.

Table 1 Effect of graphene fillers, type and content, on the tensile properties of PMMA films

\begin{tabular}{llllr}
\hline Graphene $(\%$ w/w) & 0 & $0.5($ GPMMA) & 1 (GPMMA) & $3(\mathrm{GPMMA})$ \\
\hline Young's modulus (MPa) & $18.7 \pm 0.2$ & $20.9 \pm 0.9$ & $21.8 \pm 0.6$ & $15.6 \pm 0.9$ \\
Tensile strength (MPa) & $35.5 \pm 2.2$ & $37.1 \pm 3.8$ & $42.0 \pm 2.4$ & $21.4 \pm 1.8$ \\
Elongation at break (\%) & $2.3 \pm 0.2$ & $3.5 \pm 0.8$ & $4.8 \pm 0.3$ & $0.9 \pm 0.1$
\end{tabular}




\section{Acknowledgements}

This work was co-financed by the International Iberian Nanotechnology Laboratory - Foundation of Science and Technology (FCT - Portugal) NANO/NMed-AT/0115/2007 (FCOMP-010124-FEDER-008439).

The authors P. A. A. P. Marques, Igor Bdkin and M. K. Singh would like to thank the Ciência Program, FCT. G. Gonçalves thanks the PhD grant from INL (International Iberian Nanotechnology Laboratory).

We also thank the facility RNME - Pole University of Aveiro, FCT Project REDE/1509/RME/2005.

\section{References}

1 M. J. Allen, V. C. Tung and R. B. Kaner, Chem. Rev., 2010, 110, 132

2 C. N. R. Rao, K. Biswas, K. S. Subrahmanyam and A. Govindaraj, $J$. Mater. Chem., 2009, 19, 2457.

3 V. C. Tung, M. J. Allen, Y. Yang and R. B. Kaner, Nat. Nanotechnol., $2009,4,25$.

4 B. Z. Jang and A. Zhamu, J. Mater. Sci., 2008, 43, 5092.

5 D. Li, M. B. Muller, S. Gilje, R. B. Kaner and G. G. Wallace, Nat. Nanotechnol., 2008, 3, 101.

6 S. Villar-Rodil, J. I. Paredes, A. Martinez-Alonso and J. M. D. Tascon, J. Mater. Chem., 2009, 19, 3591.

7 W. S. Hummers and R. E. Offeman, J. Am. Chem. Soc., 1958, 80, 1339.

8 N. A. Kotov, Nature, 2006, 442, 254.

9 S. Niyogi, E. Bekyarova, M. E. Itkis, J. L. McWilliams, M. A. Hamon and R. C. Haddon, J. Am. Chem. Soc., 2006, 128, 7720.

10 Y. Liang, D. Wu, X. Feng and K. Mullen, Adv. Mater., 2009, 21, 1679.

11 S. Villar-Rodil, J. I. Paredes, A. Martínez-Alonso and J. M. D. Tascón, J. Mater. Chem., 2009, 19, 3591.

12 H. Kong, C. Gao and D. Yan, J. Am. Chem. Soc., 2004, 126, 412.

13 M. Ghislandi, L. Prado, A. D. Oyerviedes, H. Wittich, K. Schulte and A. Barros-Timmons, J. Polym. Sci., Part A: Polym. Chem., 2008, 46, 3326.

14 S. H. Lee, D. R. Dreyer, J. An, A. Velamakanni, R. D. Piner, S. Park, Y. Zhu, S. O. Kim, C. W. Bielawski and R. S. Ruoff, Macromol. Rapid Commun., 2010, 31, 281.

15 F. A. Zhang, D. K. Lee and T. J. Pinnavaia, Polymer, 2009, 50, 4768.

16 M. Tanahashi, M. Hirose, J. C. Lee and K. Takeda, Polym. Adv. Technol., 2006, 17, 981.

17 H. P. Fu, R. Y. Hong, Y. J. Zhang, H. Z. Li, B. Xu, Y. Zheng and D. G. Wei, Polym. Adv. Technol., 2009, 20, 84.

18 Y. P. Zheng, Y. Zheng and R. C. Ning, Mater. Lett., 2003, 57, 2940

19 T. Naganuma and Y. Kagawa, Compos. Sci. Technol., 2002, 62, 1187.
20 R. Y. Hong, L. L. Chen, J. H. Li, H. Z. Li, Y. Zheng and J. Ding, Polym. Adv. Technol., 2007, 18, 901.

21 S. Kang, S. I. Hong, C. R. Choe, M. Park, S. Rim and J. Kim, Polymer, 2001, 42, 879.

22 T. Kotoky and S. K. Dolui, Colloid Polym. Sci., 2006, 284, 1163.

23 Y. Hong, H. P. Fu, Y. J. Zhang, L. Liu, J. Wang, H. Z. Li and Y. Zheng, J. Appl. Polym. Sci., 2007, 105, 2176.

24 R. G. Mathew, M. Y. Huh, J. M. Rhee, M. H. Lee and C. Nah, Polym. Adv. Technol., 2004, 15, 400.

25 H. Zhang, J. Han and B. Yang, Adv. Funct. Mater., 2010, 20, 1533.

26 M. Fang, K. Wang, H. Lu, Y. Yanga and S. Nutt, J. Mater. Chem., 2009, 19, 7098.

27 G. Gonçalves, P. A. A. P. Marques, C. M. Granadeiro, H. I. S. Nogueira, M. K. Singh and J. Grácio, Chem. Mater., 2009, 21, 4796.

28 D. Baskaran, Angew. Chem. Int. Ed., 2004, 43, 2138.

29 W. C. Oliver and G. M. Pharr, J. Mater. Res., 1992, 7, 1564.

30 Z. Spitalsky, D. Tasis, K. Papagelis and C. Galiotis, Prog. Polym. Res., 2010, 35, 357.

31 A. Niedermayer, Anal. Chem., 1967, 39, 953.

32 Z. Spitalskya, D. Tasisb, K. Papagelis and C. Galiotis, Prog. Polym. Sci., 2010, 35, 357.

33 Y. Yang, J. Wang, J. Zhang, J. Liu, X. Yang and H. Zhao, Langmuir, 2009, 25, 11808 .

34 M. Fang, K. Wang, H. Lu, Y. Yang and S. Nutt, J. Mater Chem., 2010, 20, 1982.

35 H. F. Yang, F. Li, C. Shan, D. Han, Q. Zhang, L. Niu and A. Ivaska, J. Mater. Chem, 2009, 19, 4632.

36 S. R. Wang, Y. Zhang, N. Abidi and L. Cabrales, Langmuir, 2009, 25, 11078.

37 Y. Ma, X. Y. Cao, X. J. Feng, Y. M. Ma and H. Zou, Polymer, 2007, 48, 7455 .

38 X. B. Fan, W. C. Peng, Y. Li, X. Y. Li, S. L. Wang, G. L. Zhang and F. B. Zhang, Adv. Mater., 2008, 20, 4490.

39 C. Lee, X. Wei, Q. Li, R. Carpick, J. W. Kysar and J. Hone, Phys. Status Solid B, 2009, 246, 2562.

40 C. Hall. in Polymer Materials. An Introduction for Technologists and Scientists, 2nd edn, Max Milian, London, 1989, p. 86.

41 K. Yusoh, J. Jin and M. Song, Prog. Org. Coat., 2010, 67, 220.

42 H. N. Dhakal, Z. Y. Zhang and M. O. W. Richardson, Polym. Test., 2006, 25, 846.

43 B. X. Yang, J. H. Shi, K. P. Pramoda and S. H. Goh, Compos. Sci. Technol., 2008, 68, 2490.

44 D. Blond, V. Barron, M. Ruether, K. P. Ryan, V. Nicolosi, W. J. Blau and J. N. Coleman, Adv. Funct. Mater., 2006, 16, 1608.

45 D. A. Dikin, S. Stankovich, E. J. Zimney, R. D. Piner, G. H. B. Dommett, G. Evmenenko, S. T. Nguyen and R. S. Ruoff, Nature, 2007, 448, 457.

46 S. Stankovich, D. A. Dikin, G. H. B. Dommett, K. M. Kohlhaas, E. J. Zimney, E. A. Stach, R. D. Piner, S. T. Nguyen and R. S. Ruoff, Nature, 2006, 442, 282.

47 M. Topsakal and S. Ciraci, Phys. Rev. B, 2010, 81, 024107.

48 T. Tallinen, J. A. Åström and J. Timonen, Nat. Mater., 2009, 8, 25. 